\title{
Researchers stick it to ticks to curb Lyme disease
}

he sunny weather of summer draws people outdoors to hike, picnic and camp. But hikers and picnickers can pick up tiny, potentially dangerous hitchhikers. While moving through vegetation or leaf litter, hikers may pick up ticks, which can hang onto clothes for minutes or hours until they have a chance to reach the flesh and embed undetected. These blood suckers, nymphal and adult ticks, can transmit Lyme disease. Lyme disease has been of growing concern in California, particularly in the northern counties, since the late $1980 \mathrm{~s}$ when reports of the disease increased.

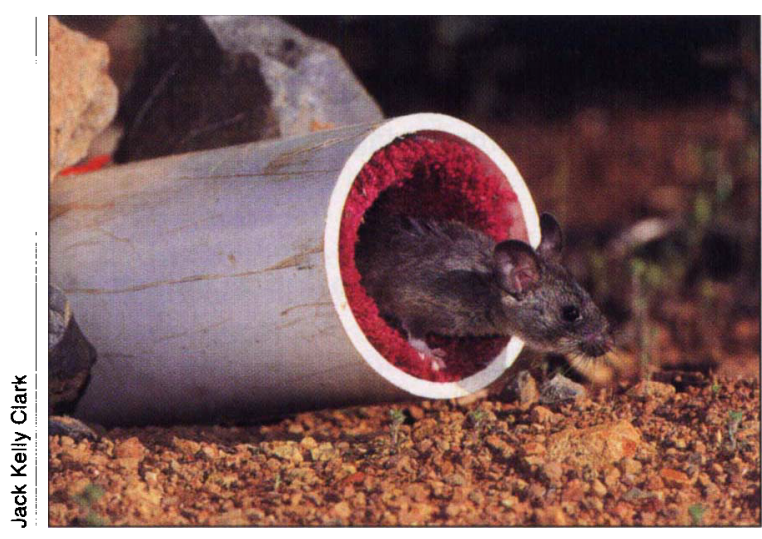

Pesticide rubs off the treated carpeting onto the wood rat as it enters the pvc pipe. The method has been very effective for reducing tick populations that may spread Lyme disease.
In California, $82 \%$ of the cases reported have been from the northern region, with the highest incidence in Mendocino and Trinity counties, says epidemiologist Robert Murray of the California Department of Health Services (CDHS). From 1991 through 1995, 24.5 cases per 100,000 were diagnosed in Mendocino County and 69 cases per 100,000 in Trinity County. The incidence was 0.5 per 100,000 people statewide. Furthermore, the infection rate is higher in some tick populations in the northern counties.

Typically less than $3 \%$ of the adult tick populations in California are infected with the spirochete, but last year UC Berkeley medical entomologist Robert Lane and James Clover of CDHS reported that $13.6 \%$ of black-legged nymphal ticks in part of Mendocino County's Potter Valley were infected.

Lyme disease, or Lyme borreliosis, is caused by a spirochetal bacterium that is transmitted to humans and animals by infected ticks. The disease got its name from being first detected in Old Lyme, Conn., in 1975. Some anecdotal and scientific evidence indicates Lyme disease has been in the United States up to 100 years.
Signs and symptoms of infection appear 3 to 32 days after an infected tick attaches and include reddish, slowly expanding rashes, fever, aches and fatigue. Without treatment, many people may suffer arthritis, heart block or nerve damage months or even years later.

It is advisable to be treated immediately with antibiotics such as tetracycline, doxycycline and amoxicillin. The sooner Lyme disease is treated, the more likely it will be cured. The good news is that the disease is not easily contracted. A person must be bitten by an infected tick that remains embedded for at least 24 to 48 hours, according to Lane, who has been studying ticks for 21 years.

Because the disease is difficult to treat at late stages, Lane has been experimenting with reducing Lyme disease by controlling ticks. In California, the western black-legged tick is primarily responsible for spreading the disease.

Using a modified version of bait tubes developed by researchers Kenneth Gage, Gary Maupin and others at the Centers for Disease Control and Prevention (CDC) in Fort Collins, Colo., Lane and his colleagues Leslie Casher, Chindi Peavey (UC Berkeley) and CDC's Joseph Piesman applied pesticides to the fur of wild rodents in California. The one-year project has been quite successful in killing or repelling the immature stages of vector ticks.

The bait tubes, which are not commercially available, are made of 20 -inch lengths of 4 -inch pvc pipe. Cracked corn, used as bait, is embedded in wax and fastened to the center of the

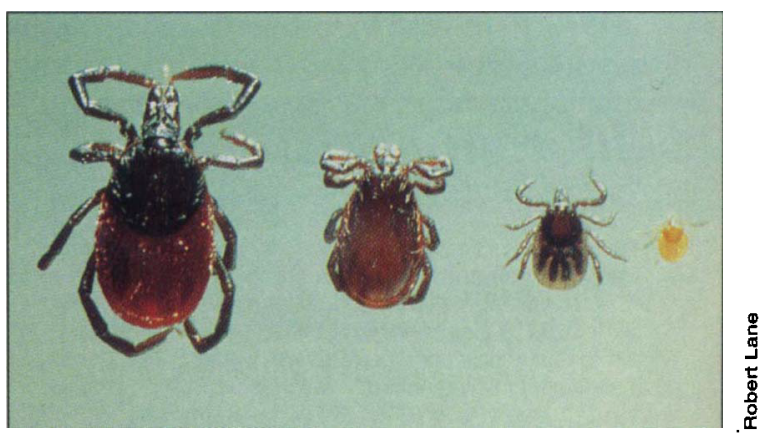

From left to right, female, male, nymph and larva of the western black-legged tick. This tick is the primary vector of the Lyme disease spirochete in California. 
pipe. Although the cracked corn is embedded in wax, animals can still smell it. It is enticing enough for a rodent to squeeze through the opening to get to the cracked corn. Inside each end of the open pipe is a ring of carpeting. The researchers squirt permethrin - a pyrethroid with low toxicity to mammals, but extreme toxicity to various insects and ticks - on the carpet. The pesticide on the carpeting is transferred to the animal's fur and any ticks and fleas in it.

The researchers placed 40 treated bait tubes near wood rat nests and 40 untreated bait tubes at another location at the UC Hopland Research and Extension Center in southern Mendocino County. Both areas had similar rodent and tick populations before the experiment.

After treatment, they found virtually no ticks on the sampling of wood rats, whereas wood rats from the untreated area were infested by one to

\section{Preventive measures}

UC Berkeley entomologist Robert Lane has written an informational sheet about tick removal and prevention of Lyme disease in California. The sheet is available free from Cooperative Extension county offices.

\section{These are a few of his tips:}

- Know how to recognize the western blacklegged tick.

- Know where the ticks live.

- Wear full-length pants and a long-sleeved shirt when in tick-infested areas.

- Treat clothing and skin with a registered tick repellent.

- Check clothing and exposed skin for ticks at least once per hour.

- Treat pets with tick repellent and check them after they have been outside.

- Recheck clothing and skin for ticks after an outing, then bathe and wash clothing.

- If you find an attached tick, remove it immediately using tweezers to grasp the tick's mouthparts as close to your skin as possible. If you don't have tweezers, use your fingers, but protect them with a tissue. Slowly and steadily pull the tick straight out. Remove any mouthparts that break off in the wound. Do not squash the tick because fluids from an infected tick may penetrate the skin.
40 ticks during different collection periods. "Control appeared to be well over $90 \%$, and on some dates it approached $100 \%$," Lane says.

In addition to killing or repelling the ticks, Lane found the insecticide freed the treated wood rats from fleas. Certain fleas also can transmit diseases, including bubonic plague, to humans. $\mathrm{CDC}$ researchers also found it effective for controlling fleas and ticks in wood rats in Colorado.

$\mathrm{He}$ is also examining another tick host - the western fence lizard, one of the most common lizards in the West. His research has shown that although ticks feed abundantly on the western fence lizard, the lizard does not become infested with Lyme disease spirochetes so it cannot serve as a source for infecting other ticks that feed upon it. He is currently investigating the biochemical basis for this phenomenon.

In previous research, Lane found that western blacklegged ticks were most likely to carry Lyme disease in the nymphal stage. In some populations of this tick, such as in Potter Valley and Berkeley's Tilden Regional Park, infection rates in nymphal ticks may exceed those in adult ticks by 3 - to 4 -fold.

One might expect infection to be twice as high in the adult ticks, so it raises the question, "Why is it so much higher in nymphs?" Lane thinks his lizard research, which he expects to publish next year, will eventually yield an answer to this paradoxical situation.

Lyme disease is not as prevalent in California as in the northeastern United States, where on average 25 to $50 \%$ of the nymphs and adults of the primary vector tick are infected with the Lyme disease spirochete. In California, the western black-legged tick has been found in 55 of the 58 counties at elevations ranging from sea level to over 7,000 feet.

Among Lane's many other tick-related projects are risk-assessment studies in recreational areas and an evaluation of the efficacy of prescribed (controlled) burning for reducing tick populations in California brushlands.
Top, Robert Lane combs the coat of a deer mouse to detect fleas. Tick treatments reduced flea populations.

Bottom, Kerry Padgett examines a western fence lizard for ticks at the UC Hopland Research and Extension Center in Mendocino County. 